\title{
Perfil Epidemiológico e Morbimortalidade dos Pacientes Submetidos à Reconstrução de Trânsito Intestinal: Experiência de um Centro Secundário do Nordeste Brasileiro
}

\author{
Epidemiologic Profile and Morbimortality of Patients Undergoing to Intestinal \\ Transit Reconstruction: \\ Experience of a Secundary Health Service in Brazil Northeast
}

JEANY BORGES E SILVA ${ }^{1}$; DJALMARIBEIRO COSTA ${ }^{1}$; FRANCISCO JULIMAR CORREIADE MENEZES ${ }^{2}$; JOSÉ MARCONITAVARES ${ }^{3}$;ADRYANO GONÇALVES MARQUES ${ }^{4}$; RODRIGODORNFELDESCALANTE ${ }^{4}$

\author{
${ }^{1}$ Residente de Cirurgia Geral do Hospital Geral Dr. Waldemar de Alcântara; ${ }^{2}$ Cirurgião Geral do Aparelho Digestivo do \\ Hospital Geral Dr. Waldemar de Alcântara; ${ }^{3}$ Cirurgião Geral, Urologista e Chefe de Residência de Cirurgia Geral do Hospital \\ Geral Dr. Waldemar de Alcântara; ${ }^{4}$ Cirurgião Geral e Coloproctologista do Hospital Geral Dr Waldemar de Alcântara.
}

\begin{abstract}
SILVA JB; COSTA DR; MENEZES FJC; TAVARES JM; MARQUES AG; ESCALANTE RD. Perfil Epidemiológico e Morbimortalidade dos Pacientes Submetidos à Reconstrução de Trânsito Intestinal: Experiência de um Centro Secundário do Nordeste Brasileiro. Rev bras Coloproct, 2010;30(3): 299-304.

RESUMO: Racional- A reconstrução do trânsito intestinal não está isenta de riscos cirúrgicos e apresenta taxas consideráveis de complicações pós-operatórias, sendo que a infecção continua a ser um dos maiores desafios existentes neste procedimento. Métodos- Foram analisados retrospectivamente 86 prontuários de pacientes com colostomia ou ileostomia, através de fatores que tivessem impacto sobre a morbimortalidade após a reconstrução de trânsito intestinal, de janeiro de 2003 a abril de 2009. Resultados- Houve 20 mulheres e 60 homens, com idade média de 43 anos. A colostomia em alça (n: 34) e o trauma abdominal indicando colostomia ou ileostomia foram as condições mais frequentes. $O$ intervalo médio entre a confecção do estoma e a reconstrução de trânsito intestinal foi 15,7 meses. O índice de morbidade foi $56,8 \%$, sendo a infecção incisional a complicação mais comum $(27.47 \%)$. A permanência hospitalar média foi 7,6 dias. Houve regressão linear positiva entre permanência hospitalar pós-operatória e a idade do paciente. Demonstrou-se associação estatisticamente significativa entre o prolongamento da permanência hospitalar e a ocorrência de complicações $(\mathbf{p}<0,001)$. Conclusão- Pode-se inferir que ocorrência de complicações pós-operatórias e idade associam-se a prolongamento da permanência hospitalar.
\end{abstract}

Descritores: Reconstrução, complicações pós-operatórias, morbimortalidade.

\section{INTRODUÇÃO}

Ostomia tem origem na palavra grega stoma, significando abertura de origem cirúrgica, quando há necessidade de desviar, temporária ou permanentemente, o trânsito normal da alimentação e/ou eliminações. Considerando-se os tipos de ostomia, a colostomia é a mais freqüente. Caracteriza-se pela exteriorização do cólon através da parede abdominal, com o objetivo de eliminação fecal. Já a abertura artificial entre o íleo, no intestino delgado, e a parede abdominal, denomina-se ileostomias. ${ }^{1}$

Desde a primeira descrição da realização de colostomia pelo francês Littré1, a utilização de estomas e suas indicações foram modificadas conforme a evolução da medicina. Sua utilização como auxílio terapêutico das afecções colorretais é bem definido, porém a carga de morbimortalidade associada à reconstrução do trânsito intestinal é ainda motivo de preocupações. ${ }^{2}$

Trabalho realizado no Serviço de Cirurgia Geral - Hospital Geral Dr. Waldemar de Alcântara - Fortaleza - CE 
A confecção de um estoma é um procedimento comum, realizado por diversas especialidades cirúrgicas, sobretudo em situações de urgência, visando à redução da morbimortalidade pós-operatória. ${ }^{3}$

A reconstrução do trânsito intestinal não está isenta de riscos cirúrgicos e apresenta taxas consideráveis de complicações pós-operatórias, sendo que a infecção continua a ser um dos maiores desafios existentes neste procedimento. ${ }^{4}$ Relatam-se na literatura taxas de morbidade variando de $0-50 \%$ e de mortalidade $0-4,5 \% .^{2}$

Assim, este artigo tem como objetivo analisar as características demográficas, a mortalidade e morbidade associada ao procedimento de reconstrução de trânsito intestinal em um ambiente de hospital secundário, relacionar a permanência hospitalar com as características epidemiológicas do paciente e com o número de complicações pós-operatórias.

\section{MÉTODOS}

Estudo retrospectivo de uma série de 86 pacientes portadores de ostomias intestinais (cólon e intestino delgado) em um ambiente de hospital secundário, a maioria encaminhados ao nosso serviço pela rede de atendimento público do estado de Ceará, no período compreendido entre janeiro de 2003 a abril de 2009.

Analisaram-se as seguintes variáveis: sexo e idade dos pacientes, a indicação e os tempos de permanência hospitalar e da ostomia, complicações pósoperatórias e óbitos.

Realizaram-se estatísticas descritiva e inferencial através dos testes Qui-quadrado de Pearson e regressão linear.

Os dados foram agrupados no programa Microsoft Office Excel $2007^{\mathrm{TM}}$ e todas as análises fo- ram realizadas pelo Medcalc v8.0 ${ }^{\mathrm{TM}}$. O nível de significância considerado foi $\mathrm{p}<0,05$.

\section{RESULTADOS}

Foi realizada análise retrospectiva de 86 prontuários de pacientes submetidos à cirurgia de reconstrução do trânsito intestinal de janeiro de 2003 a abril de 2009.

A idade média foi de 43 anos, e houve predomínio do sexo masculino $(76,74 \%)$ (Tabela 1$).$

Lesões traumáticas $(38,2 \%)$ e câncer colorretal $(22,47 \%)$ foram as indicações de ostomização mais frequentes (Tabela 2).

A perfuração por arma de fogo, com 58,82\% dos casos, foi a principal lesão traumática motivadora de ostomização (Gráfico 1).

A colostomia em alça foi o tipo de ostomia preferencialmente realizada ( $38,46 \%$ dos pacientes) (Tabela 3 )

O tempo médio de permanência com a ostomia, antes do seu fechamento, foi de 15,7 meses, com uma variação de 3 a 284 meses. $(\div 22: 278,355 \mathrm{DF}=3, \mathrm{p}<0$, 0001) (Tabela 1).

O índice de morbidade global, encontrada nesta casuística, foi de $56,80 \%(\mathrm{n}=52)$, sendo a infecção incisional a condição mórbida mais documentada, acometendo 27,47\% dos pacientes (Tabela 4).

Foram realizadas seis relaparotomias $(6,59 \%)$, sendo três por evisceração, uma por deiscência de ferida operatória, uma por hematoma de ferida e uma por estenose da anastomose, sendo submetido à nova ileostomia.

A taxa de insucesso nas reconstruções de trânsito foi de $6,59 \%$. de $9,89 \%$.

A perda de seguimento após alta hospitalar foi

Tabela 1 - Características clínico-demográficas dos 86 pacientes ostomizados.

\begin{tabular}{|c|c|c|c|c|c|c|c|}
\hline Características & & Média $\pm \mathrm{DP}$ & Mediana & Intervalo & $\mathbf{N}$ & $\%$ & $\mathbf{p}$ \\
\hline \multirow[t]{2}{*}{ Sexo } & Homem & & & & 66 & 77 & $\mathrm{P}=0,02$ \\
\hline & Mulher & & & & 20 & 23 & \\
\hline Idade & & $43 \pm 18,2$ anos & & $12-83$ anos & 86 & 100 & \\
\hline Tempo de ostomia & & $15,56 \pm 44,99$ meses & 9 meses & 3-384 meses & 71 & 77 & $\mathrm{P}<0,0001$ \\
\hline $\begin{array}{l}\text { Tempo de } \\
\text { permanência hospitalar }\end{array}$ & & $7,57 \pm 6,42$ dias & 5 dias & $2-45$ dias & 86 & 94,5 & $\mathrm{P}<0,0001$ \\
\hline
\end{tabular}

DP: desvio-padrão. N: número de casos. Teste do Qui-quadrado para distribuição da normalidade 
Tabela 2 - Causas de indicações da ostomia.

\section{$\mathbf{N}^{\circ}$ de casos}

\section{Trauma $(38,20 \%)$}

Perfuração por arma de fogo

20

Perfuração por arma branca

Trauma abdominal fechado

Trauma perineal

\section{Câncer colorretal $(22,47 \%)$}

Abdome Agudo Inflamatório (16,85\%)

Perfuração intestinal

Diverticulite

Apendicite

\section{Abdome Agudo Obstrutivo (5,62\%)}

Volvo de Sigmóide

Hérnia encarcerada

Reintervenções (4,94\%)

Deiscência de anastomose

Estenose e deiscência da anastomose

Outras $(12,35 \%)$

Síndrome de Fournier

Iatrogenia (pós-curetagem uterina)

Iatrogenia (pós anexectomia)

Proteção de anastomose

Hérnia interna

Tumor gástrico com necrose de cólon

Doença de Hirschsprung

8

4

3

4

1

3

1

2

1

1

1
Sete pacientes $(7,69 \%)$ evoluíram em pós-operatório tardio com hérnia incisional.

Não houve registro de óbito.

Os pacientes tiveram um tempo médio de permanência hospitalar no pós-operatório de 7,6 dias, variando de 2 a 45 dias $\left(\mathrm{P}<0,0001,\left(\div^{2}: 86,037 \mathrm{DF}=6\right)\right.$. (Tabela-1).

A permanência hospitalar no pós-operatório apresentou uma curva de regressão linear positiva em função da idade $(\mathrm{P}=0,001)$. (gráfico-2).

Utilizando-se do teste do Qui-quadrado, houve uma relação significativa entre a permanência hospitalar e o número de complicações $\left(\mathrm{P}<0,001, \div^{2}: 19,31\right.$ $\mathrm{DF}=1)$ (Tabela 5).

\section{DISCUSSÃO}

Em um estudo retrospectivo de 46 pacientes submetidos a fechamento de colostomia no período

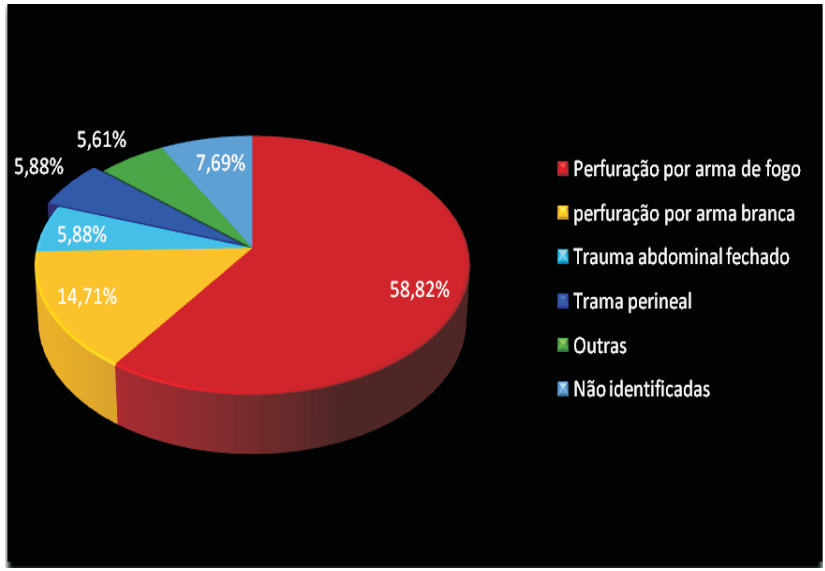

Gráfico 1 - Ostomias realizadas devido a traumas abdominais.

Março de 1988 a Abril de 1993, houve uma média de 41,8 anos, semelhante a encontrada no presente estudo, mas com predominância do sexo feminino, o que diverge desta casuística ${ }^{5}$. 
Tabela 3 - Tipos de Ostomias.

\begin{tabular}{lcc}
\hline & $\mathbf{N}^{\circ}$ de casos & \% \\
\hline Colostomia em alça & 33 & 36,26 \\
Hartmann & 27 & 29,67 \\
Ileostomia em alça & 15 & 16,4 \\
Ileostomia terminal & 7 & 7,69 \\
Mikulicz & 2 & 2,19 \\
Cecostomia & 2 & 2,19 \\
Colostomia em alça + fístula mucosa & 2 & 2,19 \\
Ileostomia em alça + hartmann & 1 & $1,10 \%$ \\
Ileostomia em alça + fistula mucosa & 1 & $1,10 \%$ \\
Hartmann + fístula mucosa & 1 & $1,10 \%$ \\
\hline
\end{tabular}

Tabela 4 - Incidência de morbidade global.

\begin{tabular}{lcr}
\hline & Número de complicações & \% \\
\hline Infecção incisional & 25 & $27,40 \%$ \\
Deiscência de ferida & 11 & $12,08 \%$ \\
Fístula êntero-cutânea & 5 & $5,49 \%$ \\
Evisceração & 3 & $3,30 \%$ \\
Abscesso intracavitário & 2 & $3,30 \%$ \\
Deiscência de anastomose & 3 & $2,20 \%$ \\
Fístula retovaginal & 1 & $1,01 \%$ \\
Hematoma de ferida & 1 & $1,01 \%$ \\
Estenose de anastomose & 1 & $1,01 \%$ \\
Total & 52 & $56,80 \%$ \\
\hline
\end{tabular}

Tabela 5 - Comparação entre o número de pacientes com complicações após reconstrução de trânsito e a permanência hospitalar.

\begin{tabular}{llccc}
\hline & & \multicolumn{2}{c}{ Complicações pós operatórias na RTI } \\
\cline { 3 - 5 } & & Sim & Não & Total \\
\hline Tempo de permanência hospitalar & $>7,6$ dias & 22 & 6 & 28 \\
& & & 43 & 58 \\
& $<7,6$ dias & 15 & 49 & 86 \\
\hline
\end{tabular}

RTI: Reconstrução de trânsito Intestinal.Teste do Qui-quadrado. $P<0,001,\left(\div^{2}: 19,31 \mathrm{DF}=1\right)$

A reconstrução do trânsito intestinal está associada a índices elevados de morbimortalidade. Bocic et al. ${ }^{6}$ estudaram 132 pacientes portadores de ileostomias e colostomias que foram submetidos à reconstrução do trânsito intestinal, relatando uma incidência de morbidade da ordem de $36,2 \%$ e mortalida- de de $1,7 \%$. Bannura et al. ${ }^{7}$, estudando 100 pacientes submetidos a reconstrução do trânsito intestinal, observaram um índice de morbidade de $34 \%$ e mortalidade de $1,7 \%$. Habr-Gama et al ${ }^{8}$, avaliando 73 pacientes submetidos à reconstrução do trânsito intestinal após colostomia a Hartmann observaram um índice de com- 


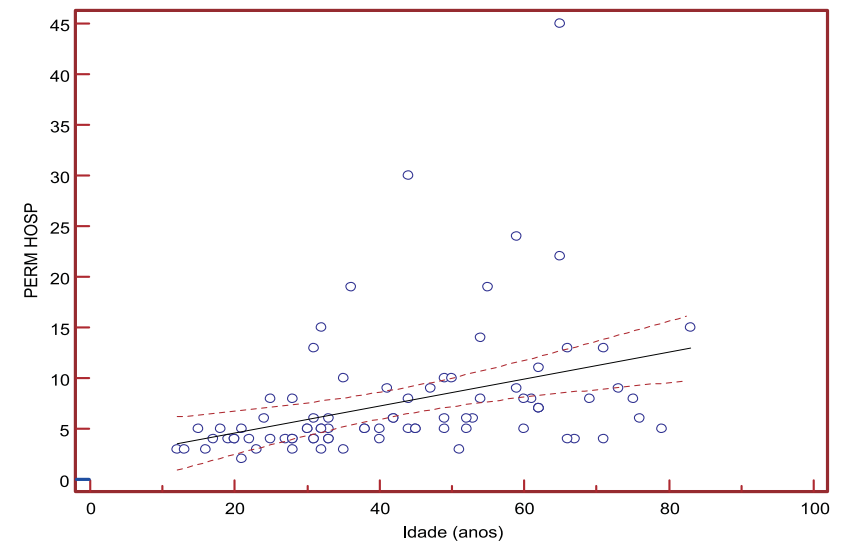

Gráfico 2 - Regressão linear da permanência hospitalar após reconstrução de trânsito intestinal em função da idade.

plicações de $34,2 \%$, sendo a infecção da ferida operatória a mais freqüente $(12,2 \%)$, e as fístulas com $8,2 \%$; sendo que a mortalidade observada nesta série foi de $3,6 \%$. Carreiro et al. ${ }^{9}$, estudando 35 pacientes portadores de colostomias em alça, após lesões traumáticas de reto e que foram submetidos ao seu fechamento, observaram uma taxa global de complicações de $25,7 \%$, sendo a infecção da ferida operatória a mais frequiente com 17,1\%; não houve óbitos nesta série. Curi et al ${ }^{10}$, avaliando 67 pacientes portadores de estomas intestinais observaram um índice de morbidade de 19,2\%, sendo o abscesso de parede $(10,4 \%)$ a complicação mais comum. Na presente casuística, observou-se uma taxa global de complicações de $56,8 \%$ após o procedimento de reconstrução do trânsito intestinal, níveis esses que poderiam ser justificados pela alta morbidade associada á ostomia pós-trauma. Em um estudo retrospectivo de 40 pacientes submetidos à colostomia pós trauma, houve uma taxa significativa de morbidade de $30 \%$ dos pacientes ${ }^{11}$.

O trauma é a causa mais comum de indicação das ostomias ${ }^{10}$, e o presente artigo corrobora com esta informação, sendo o trauma abdominal a principal causa. Houve um predomínio das colostomias em alças $(36,26 \%)$ em detrimento das ileostomias $(24,09 \%)$, apesar de haver tendência, cada vez maior, em optar-se pela ileostomia ao invés das clássicas colostomias9.

O tempo médio entre a confecção e o fechamento das ostomias foi de 15,7 meses, superior ao encontrado na literatura consultada de 6,9 meses $^{5}$. A presente casuística revelou uma regressão linear positiva estatisticamente significativa da permanência hospitalar no pós operatório de reconstrução de trânsito intestinal em função da idade, bem como uma relação também estatisticamente significativa do número de complicações com a permanência hospitalar no pósoperatório de reconstrução intestinal.

\section{CONCLUSÃO}

O procedimento de reconstrução intestinal está associado a uma alta morbidade, sendo que a ocorrência de complicações pós-operatórias e a idade dos pacientes estão diretamente relacionadas com o prolongamento do tempo de permanência hospitalar.

ABSTRACT: Background - The reconstruction of the intestinal tract is not surgical complications risk-free and is associated to postoperative complications high rates; furthermore, infection remains the hardest challenge in this procedure. Methods Retrospectively, eighty-six patients with intestinal stomas were analyzed through factors that impact on the morbimortality afterwards intestinal transit reconstruction, since January 2003 to April 2009. Results - Loop colostomy (n=34) and abdominal trauma implicating $\mathbf{3 8 . 2} \%$ of indications to colostomy or ileostomy were the most frequent conditions. The mean interval between stoma confection and intestinal transit reconstruction was 15.7 months. The morbidity frequency was $\mathbf{5 6 . 8 \%}$ and incisional infection was its commonest complication (27.47\%). The mean inpatient length of stay was 7.6 days. There was positive linear regression between post-operative inpatient length of stay and inpatient's age. Inpatient length of stay prolongation is associated to occurrence of complications $(\mathbf{p}<0,001)$. Conclusion - Post-operative complications and age are associated to inpatient length of stay prolongation.

Key words: Reconstruction, postoperative complications, morbimortality.

\section{REFERÊNCIAS}

1- Stumm EMF, Oliveira ERA, Kirschner RM. Perfil de pacientes ostomizados, Scientia Medica. 2008; 18(1)26-30.
2- Bahten LCV, Nicoluzzi JEL, Silveira F, Nicollelli, GM, Kumagai LY, De Lima VZ. Morbimortalidade da Reconstrução de Transito Intestinal Colônica em Hospital Universitário - Análise de 42 Casos. 2006; 26(2): 123-127. 
3- Santos CHM; Bezerra MM; Bezerra FMM; Paraguassú BR. Perfil do paciente ostomizado e complicações relacionadas ao estoma. Rev bras. colo-proctol. 2007. 27(1): 16-19.

4- Biondo-Simões MLP, Brenner S, Lemos R, Duck D, Rey SD. Análise das complicações pós-operatórias em decolostomias. Acta Cir Bras 2000; 15(3): 53-57.

5- Khoury DA, Beck DE, Opelka FG, Hicks TC, Timmcke AE, Gathright JB Jr. Colostomy closure. Ochsner clinic experience. Dis Colon Rectum. 1996 Jun; 39(6): 605-9.

6- Bocic GA, Jensen CB, Abedrapo MM, Garrido RC, Pérez GO, Cúeneo AZ. Colostomías e ileostomias: 8 anos de experiência clínica. Rev Hosp Clin Univ Chile 1999; 10(3): 195200.

7- Bannura GC, Perales CG, Contreras JP, Valencia CE, Melo CL. Reconstituición de tránsito intestinal luego de la operación de Hartmann: análisis de 100 pacientes. Rev Chil Cir 1999; 51(4): 359-66.

8- Habr-Gama A, Teixeira MG, Vieira MJF, Miléu LF, Laurino Neto R, Pinotti HW. Operação de Hartmann e suas conseqüências. Rev Bras Colo-proctologia 1997; 17(1): 5-10.
9- Carreiro PRL, Lázaro da Silva A, Abrantes WL. Fechamento precoce das colostomias em pacientes com trauma do reto: Um estudo prospectivo e casualizado. Rev. Coleg. Bras. Cir 2000; 27 (5): 298-304.

10- Curi A, Moreira Júnior H, Mascarenhas JCS, Moreira JPT, Almeida AC, Azevedo IF, Louza LR, Moreira H. Morbimortalidade associada à reconstrução de trânsito intestinal. - Análise de 67 casos. Rev Bras Coloproct 2002; 22(2): 88-97.

11- Berne JD, Velmahos GC, Chan LS, Asensio JA, Demetriades D. The high morbidity of colostomy closure after trauma: further support for the primary repair of colon injuries.

\section{Endereço para correspondência:}

JEANY BORGES E SILVA

Rua Homem de Melo, 933. Bloco I-2 Apto. 201

Karnak Condominium. Cambeba. Fortaleza-Ceará CEP: 60822345

E-mail: jeanyborges@yahoo.com.br 